INTERNATIONAL JOURNAL OF MULTIDISCIPLINARY RESEARCH AND ANALYSiS

ISSN(print): 2643-9840, ISSN(online): 2643-9875

Volume 04 Issue 12 December 2021

DOI: 10.47191/ijmra/v4-i12-06, Impact Factor: 6.072

Page No.- $1804-1808$

\title{
A Study of High School Students' Well-Being during the Covid-19 Pandemic
}

\author{
Sira Burana-Osot ${ }^{1}$, Smith Taechushong ${ }^{2}$, Thanapat Thutsaringkarnsakul ${ }^{3}$ \\ 1,2,3Suankularb Wittayalai School, Bangkok, Thailand
}

ABSTRACT: Due to the COVID-19 pandemic, educational practices have been disrupted in a variety of ways affecting both students and teachers. Ample evidence among many others is the fact that students are required to switch their platforms of learning, from on-site to online learning. Research conveys that this alteration has significantly affected students in various aspects such as places to study, time management, and their health. This study therefore aims to identify problems that come from online learning encountered by students themselves through the means of survey. Thus, we had collected data from 171 high school students and school leavers, in 4 categories: 1) the level of stress, 2) the level of loneliness, 3) the level of motivation, 4) the average sleep hour during the online learning period, using an online survey. The results show that there are correlations as follows, 1) positive correlation between stress and loneliness, 2) negative correlation between stress and sleep hour, and 3) negative correlation between motivation and loneliness. Screen time affects stress levels which in turn increase the loneliness of students and decrease sleep hours. This study raises concerns to educators, teachers, and parents that their children need to be understood, and appropriate support can help them overcome challenges during this difficult time.

KEYWORDS: Loneliness; Online Learning; Motivation; Sleeping Hour; Stress Level

\section{INTRODUCTION}

\section{Digital transformation of education due to the COVID-19 pandemic}

The COVID-19 pandemic has caused a significant shift in communication in a number of ways. The advancement of technology is critical to pave the way for facilitating this shift. People in all walks of life are being encouraged to use media applications that allow for real-time connection and interaction between individuals, institutions, businesses, and even countries. Of course, education is not an exception. The pandemic has recently made digital transformation in learning more practically widespread. Schools in various countries have been closed, resulting in learning activities being available entirely online (Wangwongwiroj \& Yasri, 2021). There are schools where classes are turned to synchronous meetings where teachers and students meet through online webinar tools at a certain period. However, other schools may adopt asynchronous meetings where students can access pre-recorded class materials at any time and from any location using a computer or mobile device. This mode is entirely based on self-regulated learning (Branon \& Essex, 2001).

Despite the advancement of technology-enhanced learning which is considered a great benefit, stakeholders in the educational field including teachers and learners have reported to experience challenges due to this new learning environment, ranging from psychological, social, and physiological concerns (Suppawittaya et al., 2020). School students appear to perceive a lower level of confidence in learning in online environments compared to face-to-face learning (Wangwongwiroj \& Yasri, 2021). Many report that learning performance decreases significantly as a result of remote learning from pre-recorded materials (Kang \& Im, 2013). In addition, students' attention span has shown to decrease as a result of online learning, not to mention their level of engagement in online learning that is not always positive (Bradbury, 2016). However, numerous studies attempt to point out positive sides that come about as a result of the adaptation after the global pandemic including the development of psychological maturity (Suppawittaya et al., 2021).

\section{Online learning and stress levels}

As student gatherings in most if not all schools worldwide have been prohibited due to the COVID-19 pandemic, there has been a report of acceleration of the number of online learning. Many are arranged synchronously, while others are asynchronous using 


\section{A Study of High School Students' Well-Being during the Covid-19 Pandemic}

various webinar tools and learning management systems (Wangwongwiroj \& Yasri, 2021). This transformation has led to a substantial increase in screen time using computers, laptops, tablets and/or smartphones (Mheidly et al., 2020). Research has shown that there is a positive correlation between screen time and levels of stress (Maslach \& Leiter, 2017). More specifically, increased exposure to smart devices and screens increased stress and burnout levels. While stress is a tense emotional, physical, or mental reaction brought on by social, environmental, and/or psychological factors, burnout is a mental health condition brought on by work-related stress that involves a continuous reaction to persistent interpersonal stressors. Furthermore, burnout is exacerbated by feelings of cynicism and detachment, as well as overwhelming exhaustion, leading to a sense of ineffectiveness and lack of accomplishment (Maslach \& Leiter, 2017).

\section{Online learning and loneliness}

Many people consider school to be the first step out of adolescence. It is time for people to broaden their horizons and gain advanced knowledge to lay the groundwork for their future careers. Many people consider it to be "the best years of their lives" from a social standpoint. Many people consider their university years to be the time when they met lifelong friends, life partners, and made strong academic connections. Unfortunately, students can no longer have all of those important experiences during the global pandemic. According to a survey, one in every four UK adults reported feeling lonely due to the Coronavirus pandemic (Mental Health UK, 2020). This concept of student loneliness is very similar to the concept of learning burnout, which is defined as emotional exhaustion and negative attitudes as a result of pressure to succeed and homework overload (Lin \& Huang, 2012). This concept of burnout is critical to keep in mind throughout the pandemic, as schools attempt to maintain the same educational standards during regular face-to-face teaching. Universities scrambled to make online learning identical to face-to-face learning when COVID-19 was implemented, and they may not have considered the impact of these changes on students' well-being.

\section{Online learning and motivation}

A research study has revealed how students' motivation in the form of self-efficacy varies in various learning environments. It shows that face-to-face courses can most effectively help students maintain their level of mastery experience, vicarious experience, and verbal persuasion. Online courses in the form of asynchronous meetings, on the other hand, have the potential to reduce self-efficacy in a variety of ways, including mastery experience, vicarious experience, and verbal persuasion (Wangwongwiroj \& Yasri, 2021). In face-to-face courses, the traditional learning method increases the level of mastery experience, vicarious experience, and verbal persuasion. However, this method may cause discomfort, which may have a negative impact on individuals' learning progress. This study suggests that encountering these unfavorable circumstances may help to overcome this factor. Finally, despite the fact that online courses reduce many aspects of self-efficacy, students are most at ease and confident in this mode of learning.

\section{METHODOLOGY}

This study aims to identify problems that come from online learning encountered by students themselves through the means of survey. Its specific research question is to seek out where there are statistical correlations among stress levels, motivation to study, sleep hours, and loneliness. To answer this research question, participants in Thailand, ranging from high school students to school leavers, were invited to give their 5 -likert scale responses in an online questionnaire on a voluntary basis, with their full right to withdraw from their participation in which their identity is kept autonomous and confidential. There were 171 participants in total. The convenience sampling method was implemented in this research, in which we collected data from respondents based on their availability and willingness to participate in an online survey during the COVID-19 pandemic. Before starting with their online response, the respondents were informed of the study's goal, implying their consent.

The respondents were expected to answer four questions on their stress, loneliness, motivation, and the number of hours spent on sleeping as follows. While the levels of stress, loneliness, and motivation were to be self-measured on a scale of 1-5 (indicated in Table 1), the number of hours was to be given in a whole number.

1. Can you rate your level of stress during online learning on a 1-5 scale?

2. Can you rate your level of loneliness during online learning on a 1-5 scale?

3. Can you rate your level of study motivation during online learning on a 1-5 scale?

4. How many average hours do you spend on sleeping during an online learning period? 


\section{A Study of High School Students' Well-Being during the Covid-19 Pandemic}

Table 1: measuring scale for stress, loneliness, and motivation levels

\begin{tabular}{|l|l|}
\hline scale & meaning \\
\hline 1 & Never occurs \\
\hline 2 & Occurs sometimes \\
\hline 3 & Occurs once or twice a week \\
\hline 4 & Occurs daily \\
\hline 5 & Almost always occurs \\
\hline
\end{tabular}

The relationship between two factors in this study is determined by the correlation coefficient. To interpret the accurate size of correlation, a model proposed by Schober et al. (2018) is used. According to this model, if the value of correlation coefficient is lower than 0.10 , then that value is considered to have negligible correlation. For any value that is equal to or higher than 0.10 , the scale is separated into weak, moderate, strong, and very strong positive (negative) correlation with the size between 0.10 to 0.39 , 0.40 to $0.69,0.70$ to 0.89 , and 0.90 to 1.00 , respectively.

\section{RESULT AND DISCUSSION}

According to Table 2, descriptive statistics revealed that on a 5-likert scale, the average of stress level was 3.30, indicating that the level of stress during online learning perceived by the respondents was in a relatively high state of psychological stress. Furthermore, the mean of perceived loneliness during online learning among the respondents was 3.16, showing that the students found themselves experiencing the sense of loneliness during the global pandemic. In addition, the average of motivation was 2.29 , which indicates a relatively low level of motivation to learn using online platforms. Lastly, the average number of sleep hours among the respondents was 6.54 hours per day.

Table 2: Students' stress level, loneliness, motivation, and sleep hours

\begin{tabular}{|l|l|l|}
\hline & Mean & SD \\
\hline stress & 3.30 & 1.16 \\
\hline loneliness & 3.16 & 1.36 \\
\hline motivation & 2.29 & 1.00 \\
\hline sleep hours & 6.54 & 2.17 \\
\hline
\end{tabular}

The outbreak of COVID-19 poses a disruption to the educational system worldwide by switching from on-site learning to online (García-Morales et al., 2021). Numerous negative consequences have been caused, affecting the society since the beginning of the outbreak in Wubei, China. For example, the infirm structure of the educational system, teaching and learning environment, and workloads (Zhang et al., 2020). Apart from the effect on the system itself, people in the system are also affected as well. As a study from Beltekin et al. (2020), students have been affected by the decrease in the quality of online teaching, resulting in lower performance in testing and also the motivation level. This, as discussed by the authors, is the result of the increase in stress level on the ground that environments are not preferable for studying. Moreover, the high emotional focusing, resulting in stressful emotion, has been proved to be the factor of lowering sleep hours (Sadeh et al., 2004). Stress can largely affect the intellectual, hormonal, and phycological by attacking the hypothalamic-pituitary-adreanal axis that is responsible for controlling stress while sleeping (Han et al., 2012). As in the result from our survey (Table 3), there is a weak correlation between the sleep hours during online learning and the stress level. The correlation coefficient between these 2 collected information is -0.25 . As a result, the translation from the data is that when people have more stress level living daily life, the sleeping hour decreases. This could be evidenced by the fact that stress and affect in the change in hormone balancing, such as cortisol, thyroid hormone, and also testosterone. By the change in hormonal balance, especially the sleep-related hormone, sleep deprivation can occur (Maggio et al., 2013). To conclude with this set of information, the advice could be that by changing the studying environment that would result in lower levels of stress, might increase the sleeping hours, and improve the quality of life.

Table 3: Correlation coefficients based on the questionnaire $(\mathrm{N}=171)$

\begin{tabular}{|l|l|l|l|}
\hline & loneliness & motivation & sleep hour \\
\hline stress & 0.31 & 0 & -0.25 \\
\hline loneliness & & -0.28 & 0.02 \\
\hline motivation & & & -0.02 \\
\hline
\end{tabular}




\section{A Study of High School Students' Well-Being during the Covid-19 Pandemic}

The stress and loneliness levels have a weak correlation of 0.31 . This might be due to the fact that loneliness and stress levels come from the same factor. Life quality and biological factors, such as hormones that are known to be related with stress, can lead to both stress and loneliness, and that both of them might be able to cause each other (Campagne, 2019). Stress and loneliness are closely related to each other, people with one problem usually have another too. Students who are stressed might not be able to communicate with others very well, or lack the energy to do so, making them feel lonelier. On the other hand, being lonely can cause stress. Online learning might have caused loneliness in some individuals, students can go through a full day of online classes without talking with another human. Even if they do talk, talking online cannot fulfill the experience of talking inperson (Mallen, 2003). Since humans are social animals, being lonely from online learning might cause stress due to lack of communication, which is against human nature. A study has found that the levels of stress can be increased by the increase of loneliness (Valtorta, 2015).

As for motivation to study and loneliness, the result suggests that there was a weak negative correlation $(r=0.28)$ between loneliness and motivation. To make it clearer, the more loneliness the student feels, the lower their motivation to study is. This result is similar to that of Bek (2017) as the study found that the increase in loneliness resulted in a lower number of course participation. A link between motivation and course participation can be seen in a study conducted by Formanek et al. (2019), mentioning that motivation of learners can significantly influence course engagement in a massive open online course. Not only does loneliness have an effect on students, but teachers were also negatively impacted by loneliness as a result of teaching in online classes, such as a decrease in confidence level. Importantly, their motivation to teach was negatively affected by this factor as the teachers also need to deal with stress, nervousness, and anxiety. Furthermore, the teachers felt that the engagement and motivation of students were dropped as a result of online classes during the COVID-19 pandemic (Telyani et al., 2021). The use of social networks, such as chatting with a group of friends, having online and offline interaction with family, and keeping up with the news, may help to decrease the feeling of loneliness (Taghizadeh et al., 2019). Thus, the findings in this study are in line with previous studies and suggest that loneliness might reduce motivation to study among students in this online learning era. However, there are some limitations as the causal relationship behind the two factors cannot be inferred from our study. Moreover, this study does not include the possibility in sex differences which might affect the outcomes.

\section{CONCLUSION}

Exploring 4 different factors that are affected from online learning, the causal relationships are presented through these data. It brings up 3 significant correlations that can be concluded concretely based on the information collected from 171 participants. Due to the data, there are a negative correlation between sleeping hours and stress level, a positive correlation between stress level and loneliness level, and a negative correlation between studying motivation and loneliness level. These changes of factors mostly come from the fluctuation of the hormone in the hormonal system, resulting from the longer time spent on the electrical screen. This evidence supports only some of the negative consequences posed by the educational disruption due to the outbreak of COVID-19. Researchers hope that this survey analysis would result in the improvement of online learning so that existing disadvantages could not affect the educational-related people in the future.

\section{REFERENCES}

1) Bek, H. (2017). Understanding the Effect of Loneliness on Academic Participation and Success among International University Students. Journal of Education and Practice, 8(14), 46-50.

2) Beltekin, E., \& Kuyulu, I. (2020). The Effect of Coronavirus (COVID19) Outbreak on Education Systems: Evaluation of Distance Learning System in Turkey. Journal of Education and Learning, 9(4), 1-9.i

3) Bradbury, N. A. (2016). Attention span during lectures: 8 seconds, 10 minutes, or more?. Advances in Physiology Education, 40(4), 509-513.

4) Branon, R. F., \& Essex, C. (2001). Synchronous and asynchronous communication tools in distance education. TechTrends, 45(1), 36.

5) Campagne, D. M. (2019). Stress and perceived social isolation (loneliness). Archives of gerontology and geriatrics, 82 , 192-199.

6) Formanek, M., Buxner, S., Impey, C., \& Wenger, M. (2019). Relationship between learners' motivation and course engagement in an astronomy massive open online course. Physical Review Physics Education Research, 15(2), 020140

7) García-Morales, V. J., Garrido-Moreno, A., \& Martín-Rojas, R. (2021). The transformation of higher education after the COVID disruption: Emerging challenges in an online learning scenario. Frontiers in Psychology, 12, 196.

8) Han, K. S., Kim, L., \& Shim, I. (2012). Stress and sleep disorder. Experimental neurobiology, 21(4), 141. 


\section{A Study of High School Students' Well-Being during the Covid-19 Pandemic}

9) Kang, M., \& Im, T. (2013). Factors of learner-instructor interaction which predict perceived learning outcomes in online learning environment. Journal of Computer Assisted Learning, 29(3), 292-301.

10) Lin, S. H., \& Huang, Y. C. (2012). Investigating the relationships between loneliness and learning burnout. Active Learning in Higher Education, 13(3), 231-243.

11) Maggio, M., Colizzi, E., Fisichella, A., Valenti, G., Ceresini, G., Dall'Aglio, E., ... \& Ceda, G. P. (2013). Stress hormones, sleep deprivation and cognition in older adults. Maturitas, 76(1), 22-44.

12) Mallen, M. J., Day, S. X., \& Green, M. A. (2003). Online versus face-to-face conversation: An examination of relational and discourse variables. Psychotherapy: Theory, Research, Practice, Training, 40(1-2), 155.

13) Maslach, C., \& Leiter, M. P. (2017). New insights into burnout and health care: Strategies for improving civility and alleviating burnout. Medical Teacher, 39(2), 160-163.

14) Mental Health Foundation UK (2020). People are struggling with their emotional and mental wellbeing. https://www.mentalhealth.org.uk/our-work/research/coronavirus-mental-health-pandemic/key-statistics-wave-

15) Mheidly, N., Fares, M. Y., \& Fares, J. (2020). Coping with stress and burnout associated with telecommunication and online learning. Frontiers in Public Health, 8, 672.

16) Sadeh, A., Keinan, G., \& Daon, K. (2004). Effects of stress on sleep: the moderating role of coping style. Health Psychology, 23(5), 542.

17) Schober, P., Boer, C., \& Schwarte, L. A. (2018). Correlation coefficients: appropriate use and interpretation. Anesthesia \& Analgesia, 126(5), 1763-1768.

18) Suppawittaya, P., Busarakul, T., Wangwongwiroj, T., \& Yasri, P. (2021), Psychological Adaptation after the COVID-19 Pandemic through the Lens of Evolutionary Biology. Systematic Reviews in Pharmacy, 12(3), 788-794.

19) Suppawittaya, P., Yiemphat, P., \& Yasri, P. (2021), Effects of social distancing, self-quarantine and self-isolation during the COVID-19 pandemic on people's well-being, and how to cope with it. International Journal of Science and Healthcare Research, 5(2), 12-20.

20) Taghizadeh, F., Reyhani, P., Molavi, N., Babakhanian, M., Ghazanfarpour, M., Mirzaee, F., ... \& Khorasani, F. (2019). Investigating the relationship between smartphone addiction and loneliness and its impact on motivation to progress in high school students. International Journal of Pediatrics, 7(10), 10187-10193.

21) Telyani, A. E., Farmanesh, P., \& Zargar, P. (2021). The Impact of COVID-19 Instigated Changes on Loneliness of Teachers and Motivation-Engagement of Students: A Psychological Analysis of Education Sector. Frontiers in Psychology, 4353.

22) Wangwongwiroj, T., \& Yasri, P. (2021), The Comparison of Students' Perceived Levels of Self-Efficacy in Live, Online and Live Online Courses. International Journal on Research in STEM Education, 3(1), 47-54.

23) Zhang, W., Wang, Y., Yang, L., \& Wang, C. (2020). Suspending classes without stopping learning: China's education emergency management policy in the COVID-19 outbreak. Journal of Risk and Financial Management, 13(3), 55. 